\title{
WHEN DO SOBOLEV SPACES FORM A HILBERT SCALE?
}

\author{
ANDREAS NEUBAUER
}

\author{
(Communicated by Richard R. Goldberg)
}

\begin{abstract}
In this paper we show that the usual Sobolev spaces $\left(H^{s}(\Omega)\right)_{s \in \mathbf{R}}$ are no Hilbert scale in the sense of Krein-Petunin, if $\Omega$ is an open bounded subset of $\mathbf{R}^{n}$.
\end{abstract}

1. Introduction. Let $\Omega$ be an arbitrary open subset of $\mathbf{R}^{n}$. Then the Sobolev spaces $H^{m}(\Omega)(m \in N)$ are usually defined by

$$
H^{m}(\Omega)=\left\{u \mid D^{\alpha} u \in L^{2}(\Omega) \text { for all } \alpha \text { with }|\alpha| \leq m\right\},
$$

where $D^{\alpha}=\partial^{\alpha_{1}+\cdots+\alpha_{n}} / \partial x_{1}^{\alpha_{1}} \cdots \partial x_{n}^{\alpha_{n}}, \alpha=\left\{\alpha_{1}, \ldots, \alpha_{n}\right\},|\alpha|=\alpha_{1}+\cdots+\alpha_{n}$. The derivatives $D^{\alpha} u$ are taken in the weak sense and $z \in L^{2}(\Omega)=H^{0}(\Omega)$ if and only if $z$ is measurable and

$$
\|z\|_{0}=\left(\int_{\Omega}|z|^{2} d x\right)^{1 / 2}<\infty
$$

With the norm

$$
\|u\|_{m}=\left(\sum_{|\alpha| \leq m}\left\|D^{\alpha} u\right\|_{0}^{2}\right)^{1 / 2},
$$

$H^{m}(\Omega)$ is a Hilbert space. The inner product of two elements $u, v \in H^{m}(\Omega)$ is given by

$$
(u, v)_{m}=\sum_{|\alpha| \leq m}\left(D^{\alpha} u, D^{\alpha} v\right)_{0} .
$$

For noninteger $s>0, H^{s}(\Omega)$ can be defined by interpolation, and for real $s<0$, $H^{s}(\Omega)$ is defined as the dual space of $H_{0}^{-s}(\Omega)$, the closure of $\mathscr{D}(\Omega)$ in $H^{-s}(\Omega)$, where $\mathscr{D}(\Omega)$ is the set of infinitely differentiable functions with compact support in $\Omega$ (cf., e.g., [5]).

These Sobolev spaces play an important role in the solution of boundary value problems for (elliptic) partial differential equations (cf., e.g., $[3,7])$ and in regularization of linear integral equations of the first kind with differential operators (cf. $[6, \mathbf{1}])$. In [8], Natterer proposes a new variant of Tikhonov regularization, namely Tikhonov regularization in Hilbert scales (cf. also $[\mathbf{1}, \mathbf{2}, \mathbf{9}]$ ). One advantage of this

Received by the editors April 24, 1986.

1980 Mathematics Subject Classification (1985 Revision). Primary 46E35; Secondary 35J40, $45 \mathrm{~A} 05$.

Partially supported by the Austrian Fonds zur Förderung der wissenschaftlichen Forschung (project S32/03). The author is on leave from the J. Kepler-Universität, Linz, Austria; travel support from the Fulbright Commission is gratefully acknowledged. 
approach is that one regularizes with a smooth norm, but gets convergence rates for the regularizers in a weaker norm. Another advantage is that, if the exact solution is smooth enough, one obtains higher convergence rates than with ordinary Tikhonov regularization. Hilbert scales are defined as follows (cf. [4]):

Let $L$ be a densely defined selfadjoint strictly positive operator $L$ in a Hilbert space $X$ that fulfills $\|L x\| \geq\|x\|$ on its domain. For $s \geq 0$ let $X_{s}$ be the completion of $\bigcap_{k=0}^{\infty} D\left(L^{k}\right)$ with respect to the Hilbert space norm induced by the inner product

$$
(x, y)_{s}:=\left(L^{s} x, L^{s} y\right)
$$

and for $s<0$ let $X_{s}$ be the dual space of $X_{-s}$. Then $\left(X_{s}\right)_{s \in \mathbf{R}}$ is called a Hilbert scale (induced by the operator $L$ ).

If one speaks of Hilbert scales one usually thinks of "the scale of" Sobolev spaces. It has been shown in [4] that the Sobolev spaces $H^{s}\left(\mathbf{R}^{n}\right)$ build a Hilbert scale. In this paper we show that this is no longer true for $H^{s}(\Omega)$, if $\Omega$ is an open bounded subset of $\mathbf{R}^{n}$. Moreover, we will see in $\S 2$ that Sobolev spaces with certain boundary conditions are a Hilbert scale.

2. Main result. Let $X_{1}, X_{2}$ be real Hilbert spaces with $X_{2}$ dense in $X_{1}$ and

$$
\|x\|_{1} \leq\|x\|_{2} \text { for all } x \in X_{2} \text {. }
$$

It is well known (cf., e.g., [5]) that there exists a densely defined selfadjoint strictly positive operator $L$ in $X$, with $D(L)=X_{2}$ and $\|L x\|_{1}=\|x\|_{2}$ for all $x \in X_{2}$. In the next proposition we show that there exists only one operator $L$ with these properties and determine $L$ from the embedding operator $i: X_{2} \rightarrow X_{1}$.

PROPOSITION 2.1. $L: D(L)\left(\subset X_{1}\right) \rightarrow X_{1}$ is densely defined selfadjoint and strictly positive with

$$
\|L x\|_{1} \geq\|x\|_{1} \quad \text { for all } x \in D(L)
$$

such that

$$
D(L)=X_{2} \quad \text { and } \quad(L x, L y)_{1}=(x, y)_{2} \quad \text { for all } x, y \in X_{2}
$$

if and only if

$$
L=\left(i^{*}\right)^{-1 / 2}
$$

where $i: X_{2} \rightarrow X_{1}$ is the embedding operator and $i^{*}$ is the adjoint from $X_{1} \rightarrow X_{2}$. Since $i^{*}$ is selfadjoint from $X_{1} \rightarrow X_{1},\left(i^{*}\right)^{1 / 2}$ makes sense.

PrOOF. " $\Rightarrow "$. Since $L$ is selfadjoint, $L$ is a closed operator (cf., e.g., [7]). Together with (2.2) this implies that $\mathbf{R}(L)$ is closed. Now (2.2) and the Fredholm Alternative $\left.\overline{(\mathbf{R}(L)}=N\left(L^{*}\right)^{\perp}=N(L)^{\perp}\right)$ imply that $\mathbf{R}(L)=X_{1}$. Hence, $L^{-1}: X_{1} \rightarrow$ $X_{1}$ and $\mathbf{R}\left(L^{-1}\right)=X_{2}$. With (2.1) and (2.3) we get $\left\|L^{-1} x\right\|_{1} \leq\left\|L^{-1} x\right\|_{2}=\|x\|_{1}$. Together with $\left(L^{-1} x, y\right)_{1}=\left(L^{-1} x, L L^{-1} y\right)_{1}=\left(L L^{-1} x, L^{-1} y\right)_{1}=\left(x, L^{-1} y\right)_{1}$ for all $x, y \in X_{1}$ we get that $L^{-1}$ is bounded and selfadjoint. Therefore, $L^{-2}$ is bounded and selfadjoint from $X_{1} \rightarrow X_{1}$. But for all $x \in X_{2}$

$$
\left(x, L^{-2} y\right)_{2}=\left(L x, L^{-1} y\right)_{1}=(x, y)_{1}=(i x, y)_{1}=\left(x, i^{*} y\right)_{2},
$$

and hence $L^{-2}=i^{*}$ or $L=\left(i^{*}\right)^{-1 / 2}$. 


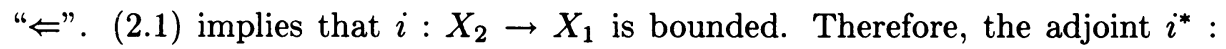
$X_{1} \rightarrow X_{2}$ is bounded. (2.1) and $\|i\|=\left\|i^{*}\right\|$ imply that

$$
\left\|i^{*} x\right\|_{1} \leq\|x\|_{1} \text { for all } x \in X_{1} .
$$

Together with

$$
\left(i^{*} x, y\right)_{1}=\left(i\left(i^{*} x\right), y\right)_{1}=\left(i^{*} x, i^{*} y\right)_{2}=\left(x, i\left(i^{*} y\right)\right)_{1}=\left(x, i^{*} y\right)_{1},
$$

this implies that $i^{*}$ is a bounded selfadjoint operator from $X_{1}$ into $X_{1}$. Since $N\left(i^{*}\right)=\overline{\mathbf{R}}(i)^{\perp}={\overline{X_{2}}}^{\perp}=X_{1}^{\perp}=\{0\}, i^{*}$ is also injective. Hence, $\left(i^{*}\right)^{1 / 2}$ is defined and also bounded selfadjoint and injective from $X_{1}$ into $X_{1}$. Now we define

$$
L:=\left(i^{*}\right)^{-1 / 2} \text { with } D(L)=\mathbf{R}\left(\left(i^{*}\right)^{1 / 2}\right) .
$$

Since $\left(i^{*}\right)^{1 / 2}$ is injective, $\overline{D(L)}=\overline{\mathbf{R}\left(\left(i^{*}\right)^{1 / 2}\right)}=N\left(\left(i^{*}\right)^{1 / 2}\right)^{\perp}=\{0\}^{\perp}=X_{1}$. This means that $D(L)$ is dense in $X_{1}$. Since $\left(i^{*}\right)^{1 / 2}$ is selfadjoint from $X_{1}$ into $X_{1}$, this implies that $L$ is a densely defined selfadjoint closed operator in $X_{1}$ (cf., e.g., [7]). Now (2.5) implies that $\left\|\left(i^{*}\right)^{1 / 2} x\right\|_{1}^{2}=\left(i^{*} x, x\right)_{1} \leq\left\|i^{*} x\right\|_{1} \cdot\|x\|_{1} \leq\|x\|_{1}^{2}$ and hence $\left\|\left(i^{*}\right)^{1 / 2} x\right\|_{1} \leq\|x\|_{1}$ for all $x \in X_{1}$. Together with (2.6) we now obtain $\|L x\|_{1} \geq\|x\|_{1}$ for all $x \in D(L)$. This means that $L$ defined by (2.6) fulfills (2.2). It remains to be shown that $L$ also fulfills (2.3).

Let now $x, y \in \mathbf{R}\left(i^{*}\right)$ and note that $\mathbf{R}\left(i^{*}\right) \subset D(L)$ and $R\left(i^{*}\right) \subset X_{2}$. Then

$$
(x, y)_{2}=\left(i^{*}\left(i^{*}\right)^{-1} x, y\right)_{2}=\left(\left(i^{*}\right)^{-1} x, i y\right)_{1}=\left(\left(i^{*}\right)^{-1 / 2} x,\left(i^{*}\right)^{-1 / 2} y\right)_{1}=(L x, L y)_{1}
$$

and hence

$$
(x, y)_{2}=(L x, L y)_{1} \quad \text { for all } x, y \in \mathbf{R}\left(i^{*}\right) .
$$

Since $L$ is closed, (2.2) implies that $\left(D(L),\|L \cdot\|_{1}\right)$ is an Hilbert space. Let $\left\{x_{n}\right\}$ be a sequence in $\mathbf{R}\left(i^{*}\right)$. Due to (2.7), $\left\{x_{n}\right\}$ is an $X_{2}$-Cauchy sequence iff $\left\{x_{n}\right\}$ is a $D(L)$-Cauchy sequence. Let now $\left\{x_{n}\right\}$ be a Cauchy sequence in both senses. Then there are unique elements $x \in X_{2}$ and $y \in D(L)$ such that $x_{n} \stackrel{X_{2}}{\rightarrow} x$ and $x_{n} \stackrel{D(L)}{\rightarrow} y$. $x_{n} \stackrel{X_{2}}{\rightarrow} x$ and (2.1) imply that $x_{n} \stackrel{X_{1}}{\rightarrow} x$. Since $L$ is closed and $\left\{L x_{n}\right\}$ is a Cauchy sequence in $X_{1}$ this implies that $x \in D(L)$ and $L x_{n} \stackrel{X_{1}}{\rightarrow} L x$, i.e., $x_{n} \stackrel{D(L)}{\rightarrow} x$ and hence $x=y$. Therefore, we have shown

$$
\text { closure of } \mathbf{R}\left(i^{*}\right) \text { in } X_{2}=\text { closure of } \mathbf{R}\left(i^{*}\right) \text { in } D(L) \text {. }
$$

Since $\left(L i^{*} x, L y\right)_{1}=\left(\left(i^{*}\right)^{1 / 2} x,\left(i^{*}\right)^{-1 / 2} y\right)_{1}=(x, y)_{1}=(x, i y)_{1}$, for all $x \in X_{1}$ and $y \in D(L), i^{*}$ is also the adjoint operator of the embedding operator $i: D(L) \rightarrow X_{1}$. Now (2.8) and the Fredholm Alternative imply that

$$
\begin{aligned}
X_{2} & =N\left(\left.i\right|_{X_{2}}\right)^{\perp}=\text { closure of } \mathbf{R}\left(i^{*}\right) \text { in } X_{2} \\
& =\text { closure of } \mathbf{R}\left(i^{*}\right) \text { in } D(L)=N\left(\left.i\right|_{D(L)}\right)^{\perp}=D(L) .
\end{aligned}
$$

Since $X_{2}=D(L)$ a continuity argument implies that (2.7) holds for all $x, y \in$ $X_{2}$.

As a consequence of Proposition 2.1 we now show that the Sobolev spaces $H^{s}(\Omega)$ are no Hilbert scale, if $\Omega$ is bounded. We first consider the case $\Omega=(0,1)$. 
COROllary 2.2. Let $m \in N$ be fixed. There exists no Hilbert scale $\left(X_{s}\right)_{s \in \mathbf{R}}$ induced by an operator $L$ defined in $L_{2}[0,1]$ such that $X_{m}=H^{m}[0,1]$ with the norm of (1.3) and $X_{s}=H^{s}[0,1]$ (with norms equivalent to that of (1.3)), if $s \neq m$, $s \geq 0$.

ProOF. Due to Proposition 2.1 there exists only one densely defined selfadjoint and strictly positive operator $L$ in $L_{2}[0,1]$ such that $D(L)=H^{m}[0,1]$ and $\|L x\|_{0}=\|x\|_{m}$ for all $x \in H^{m}[0,1]$. Therefore, we only have to show that there exists an $s>0$ such that $X_{s}:=D\left(L^{s / m}\right) \neq H^{s}[0,1]$. We will show that $X_{2 m}=D\left(L^{2}\right)=\mathbf{R}\left(i^{*}\right) \varsubsetneqq H^{2 m}[0,1]$, where $i^{*}$ is the adjoint of the embedding operator $i: H^{m}[0,1] \rightarrow L^{2}[0,1]$.

By definition of $i^{*}$, for all $x \in H^{m}[0,1]$ and $y \in L^{2}[0,1]$ we have

$$
(x, y)_{0}=\sum_{k=0}^{m}\left(x^{(k)}, i^{*} y^{(k)}\right)_{0} .
$$

Now let $z \in H^{2 m}[0,1]$. Then it follows from integration by part that

$$
\begin{aligned}
\sum_{k=0}^{m}\left(x^{(k)}, z^{(k)}\right)_{0}= & \left(x, \sum_{k=0}^{m}(-1)^{k} z^{(2 k)}\right)_{0} \\
& +\sum_{l=0}^{m-1}\left(x^{(l)}(1) \cdot B_{l} z(1)-x^{(l)}(0) \cdot B_{l} z(0)\right),
\end{aligned}
$$

where

$$
B_{l} z:=\sum_{k=0}^{m-(l+1)}(-1)^{k} z^{(2 k+l+1)}, \quad l=0,1, \ldots, m-1 .
$$

Now we show that there is a $z \in H^{2 m}[0,1]$ such that

$$
(x, y)_{0}=\sum_{k=0}^{m}\left(x^{(k)}, z^{(k)}\right)_{0} .
$$

Since $i^{*} y$ is unique in $(2.9), i^{*} y=z$. Since $H_{0}^{m}[0,1]=\left\{u \in H^{m}[0,1] \mid u^{(k)}(0)=\right.$ $\left.0=u^{(k)}(1), k=0,1, \ldots, m-1\right\}$ is dense in $L^{2}[0,1]$ (cf., e.g., [5]) (2.12) is solvable if and only if (cf. (2.10), (2.11))

$$
y=\sum_{k=0}^{m}(-1)^{k} z^{(2 k)}=: T z .
$$

From elements in $H^{m}[0,1] \backslash H_{0}^{m}[0,1]$ we derive the boundary conditions

$$
B_{l} z(0)=0=B_{l} z(1), \quad l=0,1, \ldots, m-1 .
$$

It follows from [7, Theorem 5, p. 77] that (2.13) and (2.14) define a selfadjoint differential operator $T$ in $L_{2}[0,1]$. Note that for all $z \in D(T)\|z\|_{0}^{2} \leq\|z\|_{m}^{2}=$ $(z, T z)_{0} \leq\|z\|_{0} \cdot\|T z\|_{0}$ and hence $\|z\|_{0} \leq\|T z\|_{0}$. But this implies that $\mathbf{R}(T)=$ $L_{2}[0,1]$. Therefore, we have shown that $(2.13)$ and $(2.14)$ are uniquely solvable for all $y \in L_{2}[0,1]$. Together with (2.9)-(2.12) this implies that $z=i^{*} y$ is the solution of (2.13) and (2.14). Hence,

$\mathbf{R}\left(i^{*}\right)=\left\{z \in H^{2 m}[0,1] \mid B_{l} z(0)=0=B_{l} z(1), l=0,1, \ldots, m-1\right\} \subsetneq H^{2 m}[0,1]$. 
Proposition 2.1 shows us that it is always possible to define a Hilbert scale $\left(X_{s}\right)_{s \in \mathbf{R}}$ induced by an operator $L$ in $L_{2}[0,1]$ such that $X_{m}=H^{m}[0,1]$ (for any fixed $m$ ) with the same norm. It then follows from an interpolation result (cf., e.g., [5]) that $X_{s}=H^{s}[0,1]$ with equivalent norms for all $0 \leq s \leq m$. But Corollary 2.2 shows that for $s=2 m$ this is no longer true. One can show, using interpolation theory, that $X_{s} \varsubsetneqq H^{s}[0,1]$ for all $s \geq m+\frac{1}{2}$. So $\left(H_{s}[0,1]\right)_{s \geq 0}$ is no Hilbert scale, but for any fixed $m \in N\left(H_{s}[0,1]\right)_{0 \leq s \leq m}$ is part of a Hilbert scale. In Corollary $2.2 X_{m}$ had to be equal to $H^{m}[0,1]$ with the same norm. We did not allow $X_{m}$ to have an equivalent norm. This is crucial for Tikhonov regularization in Hilbert scales, since the convergence rates results for a regularized solution of an ill-posed problem depend on the norm of the space in which one regularizes (cf., e.g., [1]).

We now show that an analogous result to Corollary 2.2 also holds for Sobolev spaces $H^{s}(\Omega)$, where $\Omega$ is an open bounded subset of $\mathbf{R}^{n},(n>1)$, with sufficiently smooth boundary. We only consider the case $m=1$.

COROLlaRY 2.3. Let $n>1$ be fixed and let $\Omega$ be an open bounded subset of $\mathbf{R}^{n}$ with $C^{1,1}$ boundary (i.e., the boundary is continuously differentiable and the first derivative is Lipschitz). Then there exists no Hilbert scale $\left(X_{s}\right)_{s \in \mathbf{R}}$ induced by an operator $L$ defined in $L_{2}(\Omega)$ such that $X_{1}=H^{1}(\Omega)$ with the norm of (1.3) and $X_{s}=H^{s}(\Omega)$ (with norms equivalent to that of (1.3)), if $s \neq 1, s \geq 0$.

PROOF. Analogously to the proof of Corollary 2.2 we only have to show that $\mathbf{R}\left(i^{*}\right) \varsubsetneqq H^{2}(\Omega)$, where $i^{*}$ is the adjoint of the embedding operator $i: H^{1}(\Omega) \rightarrow$ $L^{2}(\Omega)$. By definition of $i^{*}$, for all $x \in H^{1}(\Omega)$ and $y \in L^{2}(\Omega)$ we have

$$
(x, y)_{0}=\left(x, i^{*} y\right)_{0}+\left(\nabla x, \nabla i^{*} y\right)_{0}
$$

Now let $z \in H^{2}(\Omega)$. Since $H_{0}^{1}(\Omega)$ is dense in $L_{2}(\Omega)$ (cf. [5]), Greens identity,

$$
(\nabla x, \nabla z)_{0}=-(x, \Delta z)_{0}+\int_{\Gamma} x \frac{\partial z}{\partial v} d \sigma
$$

where $\Gamma$ is the boundary of $\Omega$ and $\partial / \partial v$ is the normal derivative, implies that $z \in H^{2}(\Omega)$ solves

$$
(x, y)=(x, z)_{0}+(\nabla x, \nabla z)_{0},
$$

if and only if

$$
y=z-\Delta z
$$

and

$$
\int_{\Gamma} x \frac{\partial z}{\partial v} d \sigma=0 \text { for all } x \in H^{1}(\Omega)
$$

The trace theorem (cf. [3, Theorem 1.5.1.2]) implies that $\left.x \rightarrow x\right|_{\Gamma}$ is surjective from $H^{1}(\Omega) \rightarrow H^{1 / 2}(\Gamma)$. Since $H^{1 / 2}(\Gamma)$ is dense in $L_{2}(\Gamma)$, this implies that $(2.19)$ is equivalent to

$$
\frac{\partial z}{\partial v}=0 \quad \text { on } \Gamma .
$$

Now [3, Theorem 2.4.2.7] implies that (2.18) together with (2.20) has a unique solution for all $y \in L_{2}(\Omega)$. Therefore, (2.15) and (2.17) imply that $\mathbf{R}\left(i^{*}\right)=\{z \in$ $\left.H^{2}(\Omega) \mid \partial z / \partial v=0\right\} \varsubsetneqq H^{2}(\Omega)$. 
Corollary 2.3 not only tells us that $\left(H^{s}(\Omega)\right)_{s \geq 0}$ is no Hilbert scale. By interpolation theory, it follows from the proof of Corollary 2.3 that $\left(H^{s}(\Omega)\right)_{0 \leq s \leq 1}$ is part of a Hilbert scale $\left(X_{s}\right)_{s \geq 0}$, where for $s>1, X_{s}$ is the Sobolev space $H^{s}(\Omega)$ with certain boundary conditions.

\section{REFERENCES}

1. H. W. Engl and A. Neubauer, Optimal discrepancy principies for the Tikhonov regularization of integral equations of the first kind, Constructive Methods for the Practical Treatment of Integral Equations, G. Hämmerlin and K. H. Hoffman (eds.), Birkhäuser, Basel, 1985, pp. 120-141.

2. _ Convergence rates for Tikhonov-regularization in finite-dimensional subspaces of Hilbert scales, Proc. Amer. Math. Soc. 102 (1988), 587-592.

3. P. Grisvard, Elliptic problems in nonsmooth domains, Monographs and Studies in Math., no. 24, Pitman, Boston, Mass., 1985.

4. S. G. Krein and J. I. Petunin, Scales of Banach spaces, Russian Math. Surveys 21 (1966), 85-160.

5. J. L. Lions and E. Magenes, Non-homogeneous boundary value problems and application. I, Springer-Verlag, Berlin and New York, 1972.

6. J. Locker and P. M. Prenter, Regularization with differential operators. I, J. Math. Anal. Appl. 74 (1980), 504-529; II: SIAM J. Numer. Anal. 17 (1980), 247-267.

7. M. A. Naimark, Linear differential operators, Part II, Harrap, London, 1968.

8. F. Natterer, Error bounds for Tikhonov regularization in Hilbert scales, Appl. Anal. 18 (1984), 29-37.

9. A. Neubauer, An a-posteriori parameter choice for Tikhonov-regularization in Hilbert scales leading to optimal convergence rates, SIAM J. Numer. Anal. (to appear).

Department of Mathematical Sciences, University of Cincinnati, CincinNATI, OHIO 45221-0025

Current address: Institut für Mathematik, Universität Linz, A-4040 Linz, Austria 